\title{
A VARIATIONAL PRINCIPLE FOR COMPLEX BOUNDARY VALUE PROBLEMS
}

\author{
ADNAN ATEF HAJJ \\ Department of Mathematics \\ U.A.E.University, A1-Ain \\ United Arab Emirates \\ (Received November 18, 1986)
}

\begin{abstract}
This paper provides a variational formalism for boundary value problems which arise in certain feilds of research such as that of electricity, where the a'ssociated boundary conditions contain complex periodic conditions. A functional is provided which embodies the boundary conditions of the problem and hence the expansion (trial) functions need not satisfy any of them.
\end{abstract}

KEY VORDS AND PHRASES Variational principle, functional, stationary, boundary conditions complex functions, line integral.

1980 AMS SUBJECT CLASSIFICATION CODE. 35A15.

\section{INTRODUCTION.}

Motivated by complex periodic boundary conditions which arise in certain problems such as those of modelling the stator of a turbogenerator (see next section for detail), we give in this paper a variational formalism which takes into consideration such boundary conditions. We produce a functional which is stationary at the solution of a given boundary value problem for a class of expansion functions which do not satisfy any of the boundary conditions; these are satisfied only at the solution point. Three types of conditions are considered: 1) Dirichlet conditions, 2) Neumann or mixed conditions and 3) periodic conditions on parallel segments of the boundary.

Let $R$ be a given complex domain with boundary $\Gamma$. Following the work of Delves and Hall [1], we split the boundary into four non-overlapping segments $\Gamma_{i} i=1,2,3,4$ and assume that periodicity conditions are imposed on the segments $\Gamma_{3}$ and $\Gamma_{4}$ such that for some fixed a, $\Gamma_{4}=\left\{\underline{y}=\underline{x}+\underline{a} \mid \underline{x} \varepsilon \Gamma_{3}\right\}$. In this case we have the relations:

$$
\underline{n}_{4}(\underline{x}+\underline{a})=\left(\underline{n}_{3} \cdot \underline{n}_{4}\right) \cdot \underline{n}_{3}(\underline{x})
$$

and

$$
\int_{\Gamma_{4}} I(\underline{y}) d s=\int_{\Gamma_{3}} I(\underline{x}+\underline{a}) d s
$$

where $\underline{n}_{3}$ and $\underline{n}_{4}$ are the unit outward normals to $\Gamma_{3}$ and $\Gamma_{4}$ respectively and $\int \mathrm{ds}$ is a 1 ine integral along the boundary with positive direction taken counterclockise.

2. THE PROBLEM

Let the problem whose solution is sought be of the following form: 


$$
-V^{2} u+b(\underline{x}) u=g(\underline{i}), \underline{x} \varepsilon ?
$$

with the prescribed boundary conditions:

$$
\begin{aligned}
u(\underline{x}) & =g_{1}(\underline{x}), \underline{x} \varepsilon \Gamma_{1} \\
\nabla u \cdot \underline{n}(\underline{x}) & =q u(\underline{x})+g_{2}(\underline{x}), \underline{x} \varepsilon \Gamma_{2} \\
u(\underline{x}) & =e^{1 \theta} u(\underline{x}+\underline{a}), \underline{x} \varepsilon \Gamma_{3} \\
\nabla u \cdot \underline{n}(\underline{x}) & =-e^{-i \theta} \nabla u \cdot \underline{n}(\underline{x}+\underline{a}), \underline{x} \varepsilon \Gamma_{3}
\end{aligned}
$$

where $\Gamma_{2}$ and/or $\Gamma_{3}$ may be vold.

In modelling the stator of a turbogenerator where the rotor rotates at angular frequency and is effectively a bar magnet generating a rotating magnetic field, periodic boundary conditions of the form:

$$
u(\underline{x})=e^{i \theta} u(\underline{x}+\underline{a})
$$

arıse for the first harmonic component; and the normal gradient condition has:

$$
\nabla \mathrm{u} \cdot \underline{\mathrm{n}}(\underline{\mathrm{x}})=-\mathrm{e}^{-\mathrm{i} \theta} \nabla \mathrm{u} \cdot \underline{\mathrm{n}}(\underline{\mathrm{x}}+\underline{\mathrm{a}})
$$

where $\theta$ is the sector angle. These tivo conditions are exactly the last two conditions o.: $(2.1 . b)$.

3. A FUNCTIONAL EMBODYING THE BOUNDARY CONDITIONS.

In this section we produce a functional which is stationary at the solution of (2.1) for a class of functions which do not satisfy any of the boundary conditions sinc? these conditions are incornorated via suitable terms in the functional $\mathrm{J}$ given as:

$$
\begin{aligned}
J(V)= & \int_{R}\left[\nabla^{2} V+B V^{2}-2 g V\right] d \underline{x} \\
& +2 \int_{\Gamma_{1}}\left(g_{1}-V\right)(\nabla V \cdot \underline{n}) d s \\
& -2 \int_{\Gamma_{2}}\left[q / 2 V^{2}+g_{2} V\right] d s \\
& -\int_{\Gamma_{3}}\left[V(\underline{x})-e^{i \theta} V(\underline{x}+\underline{a})\right]\left[\nabla V(\underline{x})-\left(\underline{n}_{3} \cdot \underline{n}_{4}\right) e^{-i \theta} \nabla V(\underline{x}+\underline{a})\right] \cdot \underline{n} d s
\end{aligned}
$$

Next, it will be shown that if we expand the trial function $V$ about the true solution $u$, of (2.1): $V=u+\varepsilon w$, where $\varepsilon$ is a scalar and $w$ is an arbitrary variation, then $J(V)$ is stationary.

Define

$$
\begin{aligned}
& G(\varepsilon)=J(u+\varepsilon w) \text {, then } \\
& \frac{\mathrm{dG}(0)}{\mathrm{d} \varepsilon}=2 \int_{R}[\nabla w \cdot \nabla u+B w u-g w] \cdot \underline{n} \underline{d} \underline{x} \\
& +2 \int_{\Gamma_{1}}\left[\left(g_{1}-u\right) \nabla w-w \nabla u\right] \cdot \underline{n} d s \\
& -2 \int_{\Gamma_{2}}\left(q u+g_{2}\right) w d s \\
& -\int_{\Gamma_{3}}\left[u(\underline{x})-e^{i \theta} u(\underline{x}+\underline{a})\right]\left[\nabla w(\underline{x})-\left(\underline{n}_{3} \cdot \underline{n}_{4}\right) e^{-1 \theta} \nabla w(\underline{x}+\underline{a})\right] \cdot \underline{n} d s \\
& -\int_{\Gamma_{3}}\left[w(\underline{x})-e^{i \theta} w(\underline{x}+\underline{a})\right]\left[\nabla u(\underline{x})-\left(\underline{n}_{3} \cdot \underline{n}_{4}\right) e^{-i \theta} \nabla u(\underline{x}+\underline{a}) \cdot \underline{n} d s\right.
\end{aligned}
$$

The first line integral in (3.2) reduces by Green's theorem and (2.1.a) to: 


$$
\begin{aligned}
2 \int_{R}[V w \cdot \nabla u+B u w-g w] d \underline{x} & =2 \int_{\Gamma} v \nabla u \cdot \underline{n} d s \\
& =\left(\int_{\Gamma_{1}}+\int_{\Gamma_{2}}+\int_{\Gamma_{3}}+\int_{\Gamma_{4}}\right)(2 w \nabla u) \cdot \underline{n} d s
\end{aligned}
$$

where we have written the line integral of (3.3) as the sum of four line integrals along the boundaries into which $\Gamma$ has been decomposed. The integrals over $\Gamma_{1}$ and $\Gamma_{2}$ of ( 3.4 ) cancel the corresponding integrals over $\Gamma_{1}$ and $\Gamma_{2}$ in (3.2) taking into consideration the boundary conditions in (2.1.b). Also from (2.1.b), it is obvious that the first of the two line integrals over $\Gamma_{3}$ in (3.2) is equal to zero. What is left is to show that the last integral in (3.2)(hereafter referred to as LI) cancels the line integrals over $\Gamma_{3}$ and $\Gamma_{4}$ in $(3.4)$. But

$$
\begin{aligned}
L I= & -\int_{\Gamma_{3}} w(\underline{x}) \nabla u(\underline{x}) \cdot \underline{n} d s \\
& -\int_{\Gamma_{3}} w(\underline{x})\left[-e^{-i \theta} \nabla u(\underline{x}+\underline{a})\right]\left(\underline{n}_{3} \cdot \underline{n}_{4}\right) \cdot \underline{n}_{3} d s \\
& -\int_{\Gamma_{3}} w(\underline{x}+\underline{a})\left[-e^{i \theta} \nabla u(\underline{x}) \cdot \underline{n}\right] d s \\
& -\int_{\Gamma_{3}} w(\underline{x}+\underline{a})[\nabla u(\underline{x}+\underline{a})]\left(\underline{n}_{3} \cdot \underline{n}_{4}\right) \cdot \underline{n}_{3} d s
\end{aligned}
$$

Using the relations (1.1) ind the boundary conditions (2.1.b), we get:

$$
L I=-2 \int_{\Gamma_{3}} w(\underline{x}) \nabla u(\underline{x}) \cdot \underline{n} d s-2 \int_{\Gamma_{4}} w(\underline{x}) \nabla u(\underline{x}) \cdot \underline{n} d s
$$

These line integrals over $\Gamma_{3}$ and $\Gamma_{4}$ cancel the corresponding ones in (3.4). Hence the functional $\mathrm{J}$ is stationary at the solution $\mathrm{u}$.

4. MATRIX SET-UP.

To descibe the matrix set-up stage, we consider for convenience and simplicity the solution of the following one dimensional problem:

$$
\left[\frac{d^{2}}{d x^{2}}+B(z x)\right] f(z x)=G(z x), \quad-1 \leq x \leq 1
$$

together with the boundary conditions:

$$
f(-z)=\alpha, f(z)=\beta
$$

where $z$ is regarded as a parameter that takes any complex value. We seek an approximate solution $f_{N}(z x)$ to $f(z x)$ of the form:

$$
\mathrm{f}_{\mathrm{N}}(\mathrm{zx})=\sum_{\mathrm{n}=1}^{\mathrm{N}} \mathrm{a}_{\mathrm{n}}(\mathrm{z}) \mathrm{h}_{\mathrm{n}}(\mathrm{x}), \quad-1 \leq \mathrm{x} \leq 1
$$

Then the problem represents a one-dimensional form of (2.1); and the functional $\mathrm{J}$ given in ( 3.1 ) reduces to:

$$
J(V)=\int_{-1}^{1}\left[\left(V^{\prime}\right)^{2}+B V^{2}-2 G V\right] d x-2[\alpha-V(-1)] V^{\prime}(-1)+2[\beta-V(1)] V^{\prime}(1)
$$

The coefficionts $a_{n}(z)$ are defined by the stationary point of $J$ (at the solution where $V$ $=\mathrm{f})$; that is, by the equations:

$$
L \underline{a}=[A+B+S] \underline{a}=\underline{G}+\underline{H}
$$


where $A, \cdots$, and $S$ aic nit matrices; - allu 1 are iv-vectors, with components:

$A_{1 j}=\int_{-1}^{1} h_{1}^{\prime} h_{j}^{\prime} d x, \quad B_{1 j}=\int_{-1}^{1} h_{i} B(z x) h_{j} d x, G_{i}=\int_{-1}^{1} h_{i} G(z x) d x$,

$S_{1 j}=h_{i}(-1) h_{j}^{\prime}(-1)+h_{j}(-1) h_{i}^{\prime}(-1)-h_{i}(1) h_{j}^{\prime}(1)-h_{j}(1) h_{i}^{\prime}(1)$,

$H_{1}=\alpha h_{i}^{\prime}(-1)-\beta h_{i}^{\prime}(1), \quad i, j=1,2, \ldots, N$.

When using global expansion functions, it is desirable for stability reasons to use orthogonal polynomials ( see Mikhlin [2]). Accordingly, in (4.2) we take

$$
\mathrm{h}_{-2}=1 ; \mathrm{h}_{-1}=\mathrm{x} ; \mathrm{h}_{\mathrm{n}}=\left(1-\mathrm{x}^{2}\right) \mathrm{T}_{\mathrm{n}}(\mathrm{x}), \mathrm{n}=0,1,2, \ldots, \mathrm{r}
$$

where $r=N-3$ and $T_{n}(x)$ is the $n$th Chebyshev polynomial of the first kind. The reason for this choice of basis is the need to handle the derivative terms in the matrix $A$ without introducing artificial singularities. To calculate the elements in (4.4.b), we expand the functions $B(z x)$ and $G(z x)$ by Chebyshev series and use Fast Fourier Techniques to approximate the expansion coefficients. Thence we relate the elements $A_{i j}, B_{i j}$ and $G_{i}$ of (4.4.b) to the coefficients of these expansions. This together with a numerical example will be considored in a suhsequent paper.

While we do not attem.pt an error analysis here, the rapidity of convergence in calculating the matrix equation (4.4) has been considered formally by Delves and Mead [3], Freeman et al [4] and Delves and Bian [5]. In these papers it is shown that a complete characterisation of the convergence of the calculation can be given in terms of an assumed structure of the matix $\mathrm{L}$ in (4.4) and the convergence of the Fourier coefficients of the right hand function $G(z x)$ in (4.1.a). Both a priori and a posterinri $+\cdots \cdot \cdots+i n n$ error estimates are provided by Delves [6] where a very similar treatment to the one given in this section is used for Frdholm integral equations and from which we take ( ignoring the a priori estimate since it contains an unknown constant):

$$
\text { A posteriori estimate: } \sim \frac{\mathrm{C}}{\mathrm{s}-1} \mathrm{~N}^{-(\mathrm{s}-1)} \sim \mathrm{Na}_{\mathrm{N}}
$$

which is a standard bound; $s=\min (p, q)$ where $p$ and $q$ depend on the differentiability of $B(z x)$ and $G(z x)$. The procedure given in this section can easily be extended to two dimesions in a straightforward manner and details are omitted.

\section{REFERENCES}

1. Delves, L.M. and Hall, C.A. An Implicit Matching Principle for Global Element Calculations, J. Inst. Maths Applics.23 (1979), 223 - 234.

2. 'ikhlin, S.G. The Numerical Performance of Variational Methods, Noordhoff, Amsterdam, 1071 .

3. Delves, L.M. and Mead, K.0. On The Convergence Rates of Variational Method. I. Asymptoticaliy Diagonal Systems, Math. Comp.25 (1971), $699-716$.

4. Freeman, T.L. and Delves, L.M. and Reid, J.K. On The Convergence Rates of Variational Methods II. Systems of Type B,C, J.Inst. Maths Applics.14 (1974), $145-157$.

5. Delves, L.M. and Bain, M. On The Cptimur Choice of Weight Functions in a Class of Variational calculations, Numer. Math.27 (1977), $209-218$. 6. Delves, L.M. A Fast Method for The Solution of Fredholm Integral Equations, I. Inst.
Maths Applics.20 (1977), 173-182. 


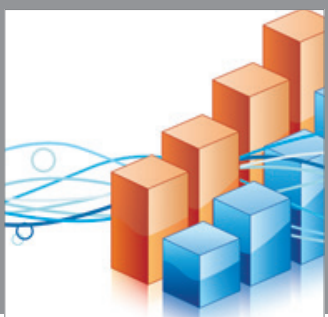

Advances in

Operations Research

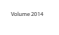

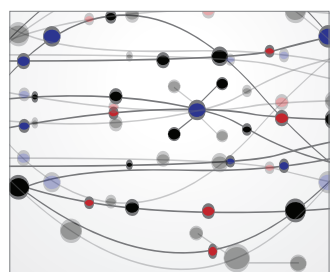

\section{The Scientific} World Journal
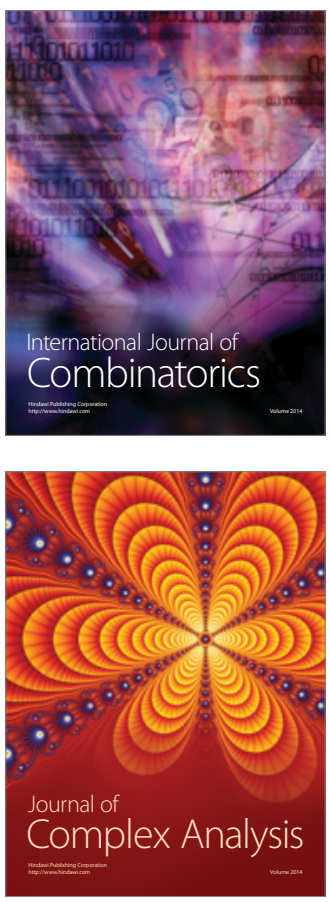

International Journal of

Mathematics and

Mathematical

Sciences
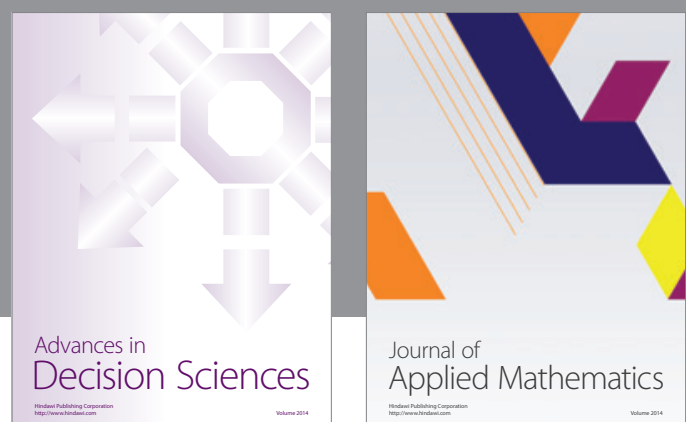

Journal of

Applied Mathematics
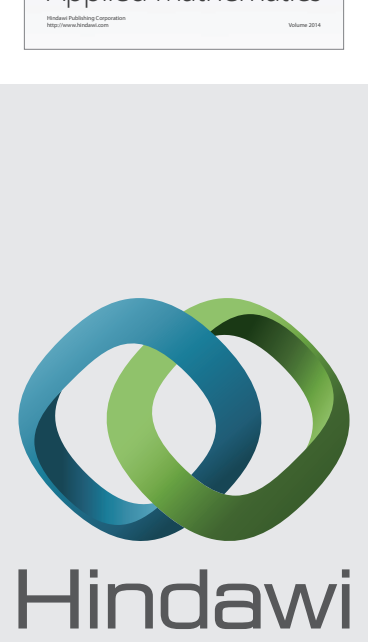

Submit your manuscripts at http://www.hindawi.com
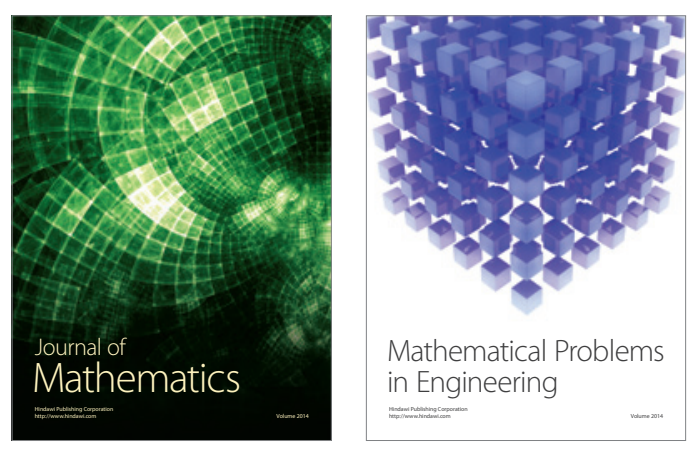

Mathematical Problems in Engineering
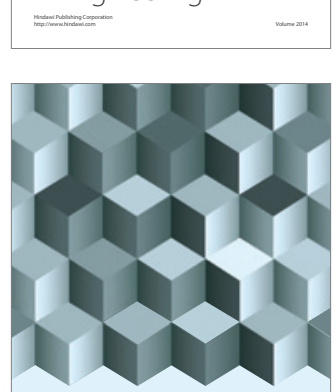

Journal of

Function Spaces
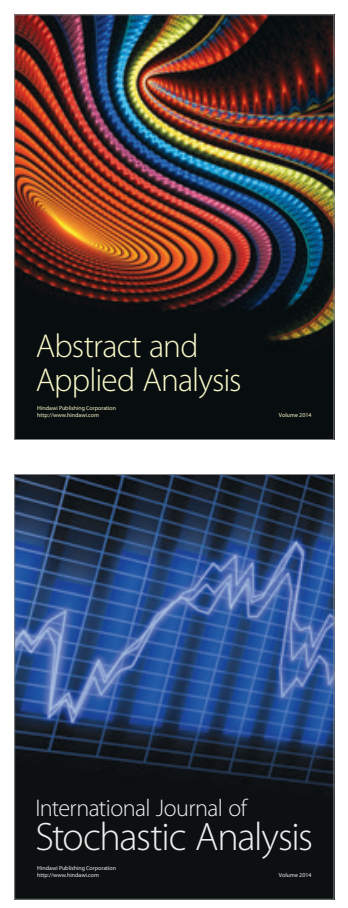

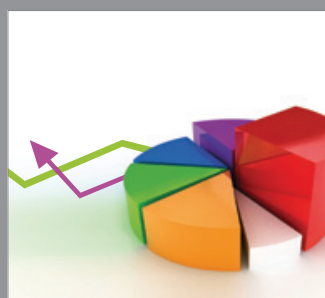

ournal of

Probability and Statistics

Promensencen
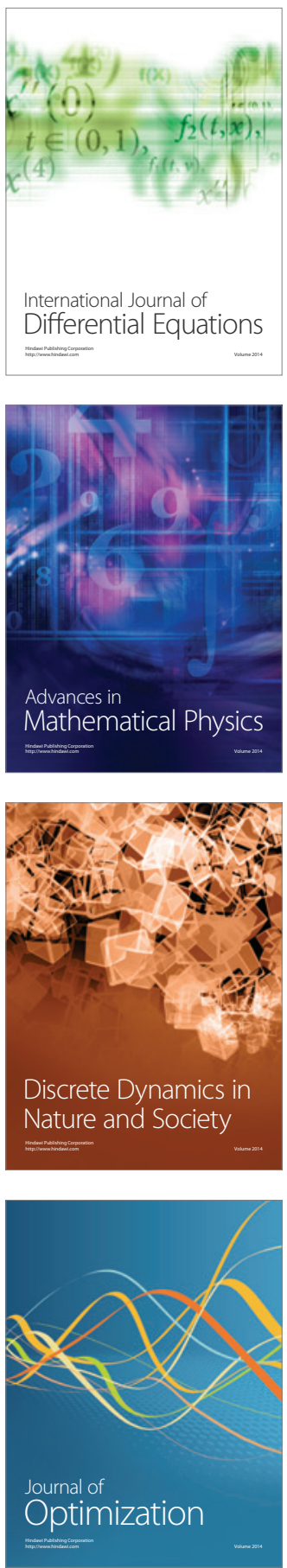